\title{
A theory of organizational structures for development and infrastructure professionals
}

This paper was downloaded from TechRxiv (https://www.techrxiv.org).

LICENSE

CC BY-NC-SA 4.0

SUBMISSION DATE / POSTED DATE

24-02-2022 / 03-03-2022

\section{CITATION}

Leite, Leonardo; Lago, Nelson; Melo, Claudia; Kon, Fabio; Meirelles, Paulo Roberto de Lima (2022): A theory of organizational structures for development and infrastructure professionals. TechRxiv. Preprint. https://doi.org/10.36227/techrxiv.19210347.v1

$\mathrm{DOI}$

10.36227/techrxiv.19210347.v1 


\title{
A theory of organizational structures for development and infrastructure professionals
}

\author{
Leonardo Leite, Nelson Lago, Claudia Melo, Fabio Kon, Paulo Meirelles
}

\begin{abstract}
DevOps and continuous delivery have impacted the organizational structures of development and infrastructure groups in software-producing organizations. Our research aims at revealing the different options adopted by the software industry to organize such groups, understanding why different organizations adopt distinct structures, and discovering how organizations handle the drawbacks of each structure. By interviewing 68 carefully-selected IT professionals and analyzing these conversations through a Grounded Theory process, we identified conditions, causes, reasons to avoid, consequences, and contingencies related to each discovered structure (segregated departments, collaborative departments, API-mediated departments, and single department). In this way, we offer a theory to explain organizational structures for development and infrastructure professionals. This theory can support practitioners and researchers in comprehending and discussing the DevOps phenomenon and its related issues, and also provides valuable input to practitioners' decision-making.
\end{abstract}

Index Terms-DevOps, software teams, organizational structures

\section{INTRODUCTION}

Over the last decade, DevOps and continuous delivery have impacted the way software-producing organizations have arranged development and infrastructure groups regarding operations activities and the setup of their underlying infrastructure ${ }^{1}$ [3]. Therefore, due to such recent changes, there is a need to investigate how these companies are structuring development and infrastructure groups regarding operations activities, such as provisioning, deployment, and monitoring.

Empirical software engineering studies have focused on identifying the different organizational structures adopted in the industry to arrange development and infrastructure professionals [4], [5], [6], [7]. However, the current literature does not reveal why these diverse organizational structures exist. This is a missing key for enabling practitioners to make good use of such results. Decision-makers in software companies are highly interested in knowing "what other companies are doing" and "why are they doing it" to support their decisions. Moreover, scholars should strive to deeply comprehend the software production phenomenon so they can adequately teach software engineering considering the reality of the industry abstracted under adequate theories. Therefore, in this paper, we focus on the following research questions:

- L. Leite, N. Lago, C. Melo, F. Kon, and P. Meirelles are with the Institute of Mathematics and Statistics of the University of São Paulo (IME-USP), São Paulo, Brazil.

E-mail: \{leofl, lago, claudia, kon, paulormm\}@ime.usp.br

1. According to the ITIL glossary [1], infrastructure refers to all of the hardware, software, networks, facilities, etc. that are required to build and operate IT services. In our context, it is helpful to add to this definition the notion that infrastructure is a layer of the software stack that can be provided as a commodity [2]. Although infrastructure must meet application non-functional requirements, it usually is provided regardless of the application domain, enabling organizations to offer it for different applications in a standardized manner.
RQ1: Why do different software organizations adopt different organizational structures regarding development and infrastructure groups?

RQ2: How do organizations handle the drawbacks of each organizational structure?

We investigate these questions in the context of softwareproducing organizations responsible for deploying the software they produce. For brevity, we refer to them in this paper simply as "organizations" or "companies."

To answer the research questions above, we conducted interviews with 68 IT professionals in 54 organizations. We analyzed these interviews by following a Grounded Theory process [8] to build a theory to explain [9] the organizational structures of development and infrastructure professionals in the context of contemporary software production.

We proceed by presenting related works in Section 2 and the research design in Section 3. Then, we present our results in Sections 4 and 5, and discuss them in Section 6. We debate our quality criteria and threats to validity in Sections 7 and 8. Finally, we draw our conclusions in Section 9.

\section{Related work}

Based on several studies [10], [11], [12], Oliveira and Takahashi summarize the basic elements of organizational structures as differentiation (division of labor) and integration (coordination) [13]. Such definition aligns well with how Conway's Law compares graphs abstracting system structures (subsystems' interconnections) and organization structures (communication paths among groups of people) [14]. Therefore, "organizational structure" (or just "structure" in this paper) can be understood as "differentiation and integration patterns".

Organizations and their structures have been extensively studied not only in the management and administration fields [15], [16], [17], [18], but also in the software engineering context. Seaman and Basili consider an organizational 
TABLE 1

Operations responsibilities and interactions in each organizational structure

\begin{tabular}{|c|c|c|c|}
\hline Organizational structure & Development differentiation & Infrastructure differentiation & Integration \\
\hline Segregated departments & $\begin{array}{l}\text { Just builds the } \\
\text { application package }\end{array}$ & $\begin{array}{l}\text { Responsible for all } \\
\text { operations activities }\end{array}$ & $\begin{array}{l}\text { Limited collaboration } \\
\text { among the groups }\end{array}$ \\
\hline Collaborating departments & $\begin{array}{l}\text { Participates/collaborates in } \\
\text { some operations activities }\end{array}$ & $\begin{array}{l}\text { Responsible for all } \\
\text { operations activities }\end{array}$ & $\begin{array}{l}\text { Intense collaboration } \\
\text { among the groups }\end{array}$ \\
\hline Single departments & $\begin{array}{l}\text { Responsible for all } \\
\text { operations activities }\end{array}$ & Does not exist & - \\
\hline API-mediated departments & $\begin{array}{l}\text { Responsible for all } \\
\text { operations activities } \\
\text { with the platform support }\end{array}$ & $\begin{array}{l}\text { Provides the platform, } \\
\text { automating much of } \\
\text { the operations activities }\end{array}$ & $\begin{array}{l}\text { Interaction happens } \\
\text { in specific situations, } \\
\text { not on a daily basis }\end{array}$ \\
\hline
\end{tabular}

structure to be a network of relationships of different types (e.g., collaborating and reporting) among developers in a company [19]. They investigate the association between organizational attributes and developers' communication efforts in code inspection meetings. Herbsleb and Roberts study how developers can optimally coordinate decisionmaking by considering the interplay among multiple decisions [20]. Nagappan et al. provide eight metrics to quantify organizational complexity, seeking to associate such metrics with software quality [21]. However, these earlier works focus on development activities, without considering infrastructure or operations concerns.

More recent works have proposed taxonomies [22] for the interactions between development and infrastructure professionals [4], [5], [6], [7], [23], [24], [25]. However, most of them do not focus on the conception of their taxonomies and instead take them as a starting point for their work. Nonetheless, Shahin et al. [5] systematically conducted interviews and surveys, and found four types of team structures: i) separate Dev and Ops teams with higher collaboration, ii) separate Dev and Ops teams with a facilitator in the middle; iii) small Ops team and more responsibilities for the Dev team, and iv) no visible Ops team.

Independently from Shahin et al., in our previous work [26], we created a taxonomy of structures in use by the industry to organize development and infrastructure professionals, which mostly coincides with that of Shahin et al. In particular, a significant difference between our taxonomy and theirs is that we identified the platform teams, a pattern recently advocated by practitioners [24], [27]. Thus, in our preliminary results [3], [26], [28], [29], we identified the following structures (which we renamed in this paper, as described in Sections 3.3 and 4):

- Segregated departments (originally "siloed departments"), with highly bureaucratized cooperation among development and operations.

- Collaborating departments (originally "classical DevOps"), focusing on facilitated communication and collaboration among development and operations.

- API-mediated departments (originally "platform teams"), in which the infrastructure team provides highly-automated infrastructure services to assist developers.

- Single departments (originally "cross-functional teams"), in which teams take responsibility for both software development and infrastructure management.

We presented such structures as a grounded theory [8] in the taxonomy form (i.e., as a classification system) [22]. We observed that professionals may be organized differently for different deployable units and that an organization can be in a transitional state from one structure to another. We also found evidence that API-mediated departments promote better delivery performance [30]. Table 1 summarizes, for each structure, (i) the differentiation between development and infrastructure groups regarding operations activities (deployment, infrastructure setup, and service operation in run-time); and (ii) how these groups interact (integration).

More on the differences between our taxonomy and those of others are published elsewhere [26]. However, Lopez-Fernandez et al. developed a taxonomy concurrently with ours [6]. Although they organize their taxonomy differently from ours, both results present essential common elements, encompassing the ideas of collaborating departments, cross-functional teams, and the provisioning of platforms. They also corroborate our finding that platform teams foster delivery performance. Lopez-Fernandez et al. [6] acknowledge the common points among the works of Shahin et al. [5], ours [26], and theirs, highlighting different insights brought by them and stressing how these models can be appreciated in conjunction, providing data and methods triangulation.

However, the above related works present taxonomies to describe structures. They do not explain why different companies adopt different organizational structures, which is our primary research goal in this paper. Nonetheless, Erich et al. [31] authored an earlier article closer to this goal, in which they seek to provide an explanation for DevOps. For six interviewed organizations, they explored: why and how to adopt DevOps, and the consequences (problems and results) of adopting it. They also explored organizational structures (e.g., discussing DevOps teams and the distribution of responsibilities). Still, their analysis is targeted at specific organizations, with no theory building. In other words, they do not provide a taxonomy of DevOps structures, but simply describe the DevOps structures of six organizations. Moreover, causes and consequences are related to the "DevOps adoption" category, not to different structures, which we discuss here.

\section{Methodology and Research design}

We applied classic Grounded Theory (GT) [8], [32] to build a theory based on data retrieved from semi-structured interviews conducted in real-world organizations. GT is a methodology originated in social sciences, but its use in the 
TABLE 2

Description of participants and organizations

\begin{tabular}{|c|c|c|c|c|}
\hline Revisit & Organizational structure & $\begin{array}{l}\text { Number of employees } \\
\text { in the organization }\end{array}$ & $\begin{array}{l}\text { Reference codes and } \\
\text { roles of interviewee }\end{array}$ & $\begin{array}{l}\text { Interviewee } \\
\text { location }\end{array}$ \\
\hline No & Single depart. & $>1000$ & I38) Developer & USA \\
\hline No & Segregated to collaborating depart. & $>1000$ & I39) Developer & Brazil \\
\hline Yes & Segregated to API-mediated depart. & $>1000$ & $\begin{array}{l}\text { I40) Developer } \\
\text { I41) Infrastructure manager }\end{array}$ & Brazil \\
\hline Yes & Collaborating depart. & {$[200,1000]$} & $\begin{array}{l}\text { I42) Infrastructure manager } \\
\text { I43) Infrastructure engineer }\end{array}$ & USA \\
\hline No & API-mediated depart. & {$[200,1000]$} & I44) Development manager & Brazil \\
\hline No & Single depart. & $<200$ & I45) Developer & Brazil \\
\hline Yes & API-mediated depart. & $>1000$ & I46) Development manager & Brazil \\
\hline No & Single depart. & $<200$ & I47) Development manager & Brazil \\
\hline Yes & API-mediated depart. & {$[200,1000]$} & $\begin{array}{l}\text { I48) Infrastructure manager } \\
\text { I49) Development manager }\end{array}$ & Spain \\
\hline No & Collaborating depart. & {$[200,1000]$} & I50) Developer & Brazil \\
\hline Yes & Segregated to collaborating depart. & {$[200,1000]$} & I51) Infrastructure engineer & Brazil \\
\hline No & Collaborating depart. & $>1000$ & $\begin{array}{l}\text { I52) Infrastructure manager } \\
\text { I54) Development manager }\end{array}$ & Brazil \\
\hline No & API-mediated depart. & {$[200,1000]$} & I53) Infrastructure manager & Brazil \\
\hline Yes & Segregated to API-mediated depart. & $>1000$ & I55) Infrastructure manager & Brazil \\
\hline No & API-mediated depart. & $>1000$ & I56) Consultant & Brazil \\
\hline No & Single depart. & $>1000$ & I57) Infrastructure engineer & Brazil \\
\hline Yes & Single depart. & {$[200,1000]$} & I58) Infrastructure manager & Brazil \\
\hline No & Collaborating to API-mediated depart. & $<200$ & I59) Infrastructure manager & Brazil \\
\hline No & Collaborating depart. & $<200$ & I60) Developer & Brazil \\
\hline No & API-mediated depart. & {$[200,1000]$} & $\begin{array}{l}\text { I61) Infrastructure manager } \\
\text { I62) Infrastructure manager }\end{array}$ & Brazil \\
\hline No & Single to API-mediated depart. & $<200$ & I63) Development manager & USA \\
\hline No & Single depart. & $<200$ & I64) CTO & USA \\
\hline No & Collaborating to single depart. & {$[200,1000]$} & $\begin{array}{l}\text { I65) Developer } \\
\text { I67) Infrastructure engineer }\end{array}$ & Brazil \\
\hline No & Segregated to API-mediated depart. & $>1000$ & I66) Architecture manager & Brazil \\
\hline Yes & Collaborating depart. & $>1000$ & I68) Development manager & Brazil \\
\hline
\end{tabular}

software engineering field is already commonplace [6], [7], [32], [33]. GT supports researchers to build theories based typically on qualitative data. We can also categorize our study as a field study [34], a category of knowledge-seeking studies, in opposition to solution-seeking studies [34]. This means that, rather than a straightforward guide to action, our theory aims to guide reasoning.

We segmented our research into two phases: the first focuses on developing an initial taxonomy (already published [26]), and the second focuses on answering RQ1 and RQ2 (the present paper). In the first phase, after initial brainstorming sessions with seven highly-skilled DevOps specialists, we interviewed 37 IT professionals of different roles and experience, working in companies of different countries, sizes, and domains. We analyzed these interviews to discover the different organizational structures used by the industry to organize development and infrastructure professionals. We analyzed such semi-structured interviews with an open coding process until we reached theoretical saturation [8]. We also got some feedback from participants on our emergent structures through online surveys. Thus, in the remainder of this section, we present the design of our second research phase.

\subsection{Sample}

To understand "why different organizations adopt different structures" (RQ1), it is crucial to analyze organizations adopting different structures. Therefore, we selected com- panies already visited by us in the first research phase [26] to make sure that we would get a good coverage of all the different structures. Since company size is expected to be a relevant factor regarding our research question [5], [26], we also aimed to select organizations of different sizes. To accomplish that, we:

- Considered transitional situations as distinctive structures (e.g., collaborating departments is one structure, while transitioning from collaborating to APImediated departments is another one).

- Grouped the visited organizations by structure and size (small, medium, large).

- Discarded the groups (structure and size) with only one member (less relevant groups).

- Aimed to visit one company from each remaining group.

Such a selection strategy left us with the goal to revisit 11 organizations within the following groups: large collaborating departments, medium collaborating departments, small collaborating departments, medium single departments, small single departments, large API-mediated departments, medium API-mediated departments, large segregated departments, small segregated departments, medium segregated departments transitioning to collaborating departments, and large segregated departments transitioning to API-mediated departments.

Considering our need for increasing generalizability, we also visited new organizations. Since it was not possible to 
know in advance what a company's structure would be, we selected them taking into account only their sizes. We did not preestablish a target on the number of new organizations to be visited - interviews continued until we reached saturation (see Section 3.6). As a form of triangulation, we also tried to interview a developer and an infrastructure professional from each organization, whenever it was applicable and possible.

Nonetheless, we note that having access to IT professionals willing to expose organizations' internal affairs can not be guaranteed in advance. Therefore, although the above strategy guided our selection, we could not strictly stick to it. In this way, Table 2 presents the new 31 carried interviews, which extend the previous 37 interviews to the total of 68 interviews we analyzed in our research. For each interview/interviewee, we also assigned an identifier (e.g., I38) to reference in this text. We conducted these interviews from May 2020 to October 2021, and they took from 20 to 63 minutes (median of 35 minutes).

The interviewees worked in the following business domains: education, health, logistic, telecommunications, public administration, development support, hiring, marketplace, travel, manufacturing, finances, mobility, games, defense, networking, semiconductors, IoT, and cloud computing. One interviewed company was a tech giant, while three were unicorns.

Although we did not plan to balance the structures among new companies, in the end, our sample was reasonably balanced. From 17 new companies, four presented the collaborating departments structure, six showed the single department structure, and seven had an API-mediated structure. For this counting, we considered a company transitioning from structure $\mathrm{X}$ to $\mathrm{Y}$ as in $\mathrm{Y}$. In particular, all the interviewed companies with segregated departments were already transitioning to some other structure.

\subsection{Interview procedure}

In the conducted semi-structured interviews, after presenting our taxonomy, we approached: which was the organizational structure in the context of the interviewee according to their opinion; why the organization adopted such a structure over the others; whether another structure would be more suitable for the interviewee context; what were the perceived (or expected) disadvantages of the discussed structures; and what were the strategies to handle such disadvantages. During interviews, we followed Adams's guidelines [35]. We provide our complete script with the rationale of each question as supplementary material.

\subsection{Resonance analysis}

Initially, 37 semi-structured interviews provided us with enough data to build the first version of our taxonomy of organizational structures. In such conversations, we employed second-level questions [36] to avoid exposing the emerging structures to interviewees. After this, we performed a brief and limited member check [37] through online surveys. The following and necessary step was to observe the use of our taxonomy in practice, verifying whether it achieves the goal of a classification (support reasoning by increasing users' cognitive efficiency [22]) and serves as a grounded theory (being applicable by practitioners [8]). To answer RQ1 and $\mathrm{RQ} 2$, we set up a favorable context to apply and refine our taxonomy - we had to use its concepts during interviews on the second research phase (Section 3.2).

We refer to the taxonomy refinement process as resonance analysis, where "resonance" alludes to the degree to which findings are understandable to participants [22]. By assessing resonance in the Grounded Theory context, we do not aim to determine whether the taxonomy is "valid" or not. Under GT principles, when faced with new conflicting evidence (e.g., a taxonomy misuse), we adapted and evolved our theory, strengthening it. We note that social theories are rarely confirmed but are instead corroborated, confronted, or evolved by new studies [38], [39], [40]. Steinmacher, for example, gathered qualitative data to refine his grounded theory with additions, deletions, and reorganizations in his model [41]. Similarly, our process guided us on managing such operations in our taxonomy: adding, deleting, reorganizing, and renaming its high-level elements.

For each interview, we coded relevant excerpts as:

- providing support to our theory, when the interviewee employs the terms of the taxonomy to discuss reality or possibilities in a precise and confident way; or

- displaying confusion about our theory, when the interviewee employs a term from the taxonomy in a different way than we would expect, or there is difficulty in selecting a term to describe its reality or possibilities.

For each excerpt coded as confusion, we defined an action to handle the situation or justify our choice of not taking any action. In consequence, the taxonomy evolved with new versions. We did not wait for interviews to be over to apply such actions; we interleaved collection, analysis, and actions, following GT principles. In this way, we conducted the last interviews based on the latest taxonomy version.

The procedures of our resonance analysis combine aspects of directed content analysis (coding by using predefined categories) and summative content analysis (counting codes and assessing the contexts for confusions) [42]. We present the results of the resonance analysis in Section 4.

\section{4 $6 \mathrm{C}$ analysis}

Glaser proposed 18 theoretical coding families to guide researchers in labeling data at the conceptual level [43]. These families were intended to sensitize these professionals to the many ways through which a concept could be examined. The "bread and butter" coding family for sociologists are what he labels "The Six C's: causes, contexts, contingencies, consequences, covariances, and conditions" [44]. Other GT studies on software engineering also use this practice [33], [45], [46].

The same author states that theoretical codes are vital because they potentiate a theory's explanatory power and increase its completeness and relevance, resulting in a grounded theory with a greater scope and parsimony [47]. Hernandez explains that "without theoretical codes, the substantive codes become mere themes to describe (rather than explain) a substantive area; the descriptive thematic approach is characteristic of qualitative research methods such as phenomenology or ethnography but not Classic 
GT" [48]. Indeed, in this paper, we present not just a "theory for analyzing," but a "theory for explaining" [9].

As it happened with Hoda et al. [45], when comparing theoretical coding families, it became evident that the 6C coding family is the most suited for our research goals. We understand that our research questions can be answered in the $6 \mathrm{C}$ format since, for a given context and certain conditions, there are causes that lead organizations to take actions expecting specific consequences - although contingencies emerge as well. Moreover, there may be a covariance of such causes, consequences, and contingencies in specific contexts and conditions.

Thus, we transcribed each interview, highlighted key points from the interviews (the excerpts with potential theoretical interest), extracted codes from the key points, and associated them with $6 \mathrm{C}$ labels. Usually, theoretical coding associates codes with the core category of the study. In our case, the four organizational structures of our taxonomy are our core categories. As the coding process advanced, we merged and abstracted different codes, as it is common to open coding too. Hence, a code may have different sources (interviews). We provide the key points and the extracted codes as supplementary material.

Although the original 6C labels provide a robust analysis framework, there is no reason to force relevant codes to fit into them. Therefore, as we perceived a need during analysis, and as agreed in review sessions, we adapted the labels - such flexibility agrees with Glaser's ideas [48]. Thus, the employed definitions of the labels used during our $6 \mathrm{C}$ analysis were:

- Characterization: identifying structures' characteristics, i.e., aspects that enable relating companies to structures.

- Conditions: environmental conditions that are necessary to implement a structure (i.e., prerequisites).

- Causes: reasons/motivations/opportunities that led the organization to adopt a particular structure and not another.

- Avoidance reasons: reasons/motivations that led the organization not to adopt a particular structure.

- Consequences: outcomes that happen or are expected to happen after an organization adopts a structure, including unexpected issues.

- Contingencies: strategies to overcome a structure's drawbacks.

We did not analyze context and covariance interview-byinterview. The context of our interviews is the one presented in Section 3.1. We performed the covariance analysis after the last interview. We used characterization codes to link interviews and their codes to structures. Nevertheless, as they largely overlap with our taxonomy's core categories [26], we do not explicitly report them here.

It is troublesome to differentiate facts from interviewees' opinions. We mitigated this issue by reporting only codes supported by at least three interviews. The rationale is that an idea supported by three persons, thus in at least two companies, is worthy of consideration to some degree. We call the conditions, causes, avoidance reasons, consequences, and contingencies supported by at least three interviews as strong codes. We present the results of the $6 \mathrm{C}$ analysis in Section 5.

\subsection{Review process}

The first author of this paper conducted the initial analysis of each interview. Periodically, other two authors joined for review sessions, in which we held discussions until reaching consensus. While the first author is also a software developer, it is relevant to note that one of the reviewer authors is also an experienced infrastructure professional. Receiving insight from this type of professional for a DevOps-related study was a recommendation given by an interviewee on the first research phase, aiming to soften possible biases given the first author's metier. We held 14 review sessions, from January to October 2021, with most of the sessions taking around two hours.

\subsection{Theoretical saturation}

At each analysis snapshot (an interview analysis or a review session), we tracked a few metrics to identify theoretical saturation [8]. For the $6 \mathrm{C}$ analysis, we defined the following metrics: number of codes, number of strong codes, and conceptual density.

The number of codes indicates the total "amount of learning" we had in each interview. Although we can always learn something new from new people, we expect diminishing returns in this metric as we approach theoretical saturation. Strong codes are supported by at least three interviews, excluding characterization codes. We also expected this metric to initially grow with new interviews and then stabilize when reaching saturation.

Each code has a support number, that is, the number of sources supporting that code. The conceptual density is the sum of support numbers divided by the total number of codes. The idea is that a high density indicates that codes are supported by multiple interviews, pointing to robust results. The expectation was that the value of this metric should only grow.

As observable in Figure 1, along with the interviews, the metrics followed the expected trends, indicating enough theoretical saturation. In detail, we note that the decreases in the number of codes (L1) are related to the review sessions, in which we deleted codes we judged to be inadequate, besides merging other ones, forming more abstract codes.

For the resonance analysis, we defined two metrics: support level and taxonomy changes. The support level is the difference between the number of support codes and the number of confusion codes. A high support level indicates a good resonance of the taxonomy with participants. This metric should ideally only grow.

Taxonomy changes is the number of changes in the high-level view of our taxonomy: addition, renaming, and removal of elements. We derived these changes from observed confusions in the interviews or even neutral comments pointing to potential improvements. We expected an initial increase in the number of changes (after all, updating the taxonomy based on the practitioners' views was in our process), followed by stabilization, indicating that the previous modifications subsequently caused fewer confusions among the interviewees. As observable in Figure 2, the metrics again followed the expected trends, indicating enough theoretical saturation. 


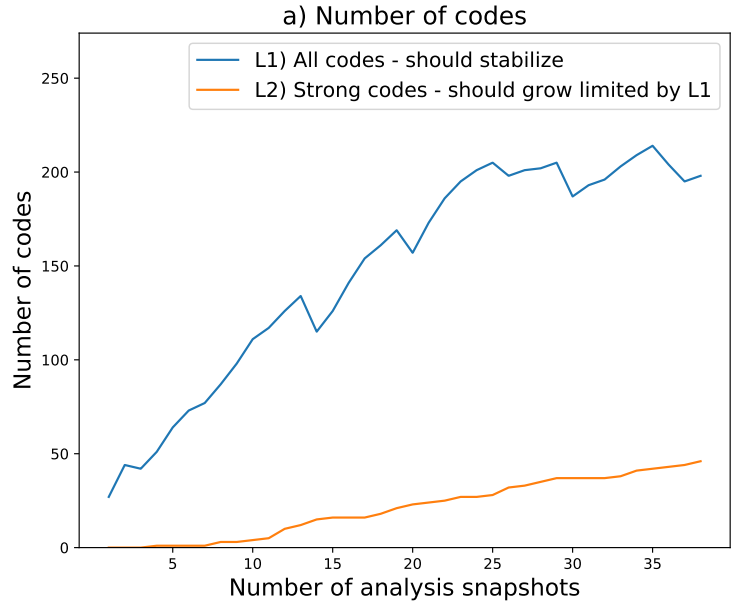

b) Conceptual density

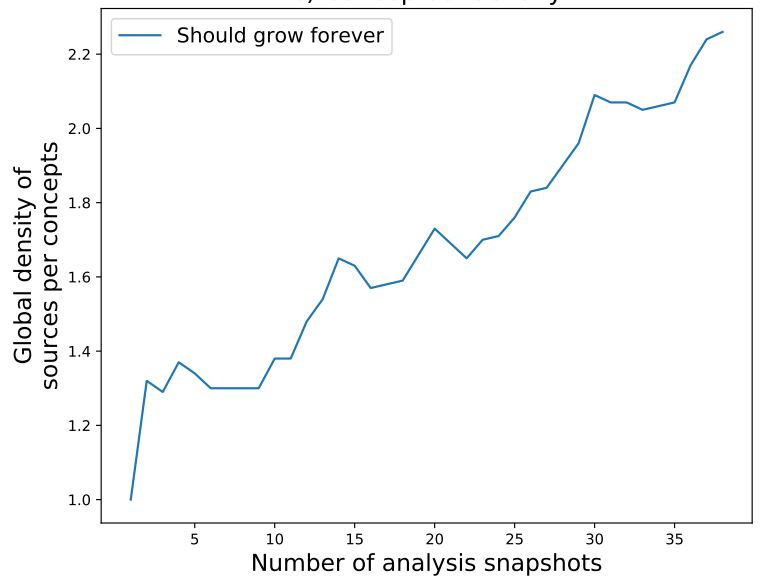

Fig. 1. Metrics of theoretical saturation for the $6 \mathrm{C}$ analysis

We clarify that the metrics used here were intuitively conceived by us, given the lack of metrics to detect theoretical saturation in Grounded Theory studies [45], [46], [49].

\section{IDENTIFYING ORGANIZATIONAL STRUCTURES}

Based on the first 37 semi-structured interviews and following GT guidelines, we elaborated an initial version of our taxonomy, with each structure having core and supplementary properties. Core properties are expected to be found in corporations with a given structure. Supplementary properties refine the explanation of a structure, but their association with organizations is not compulsory. Such properties are presented in detail elsewhere [26].

After having this initial version and more 31 semistructure interviews, we conducted our resonance analysis process, in which we coded fragments of these 31 conversations as support or confusion fragments. For each excerpt coded as confusion, we defined an action to handle the situation or justified our choice of not taking any action. In total, we recorded 35 actions, 26 of them classified as change the taxonomy, five as improve interview presentation, and four as improve report. Actions changed the high-level elements of our taxonomy (organizational structures and supplementary properties) with additions, removals, and renamings. We considered interview presentation improvements in how we explained the taxonomy to the subsequent
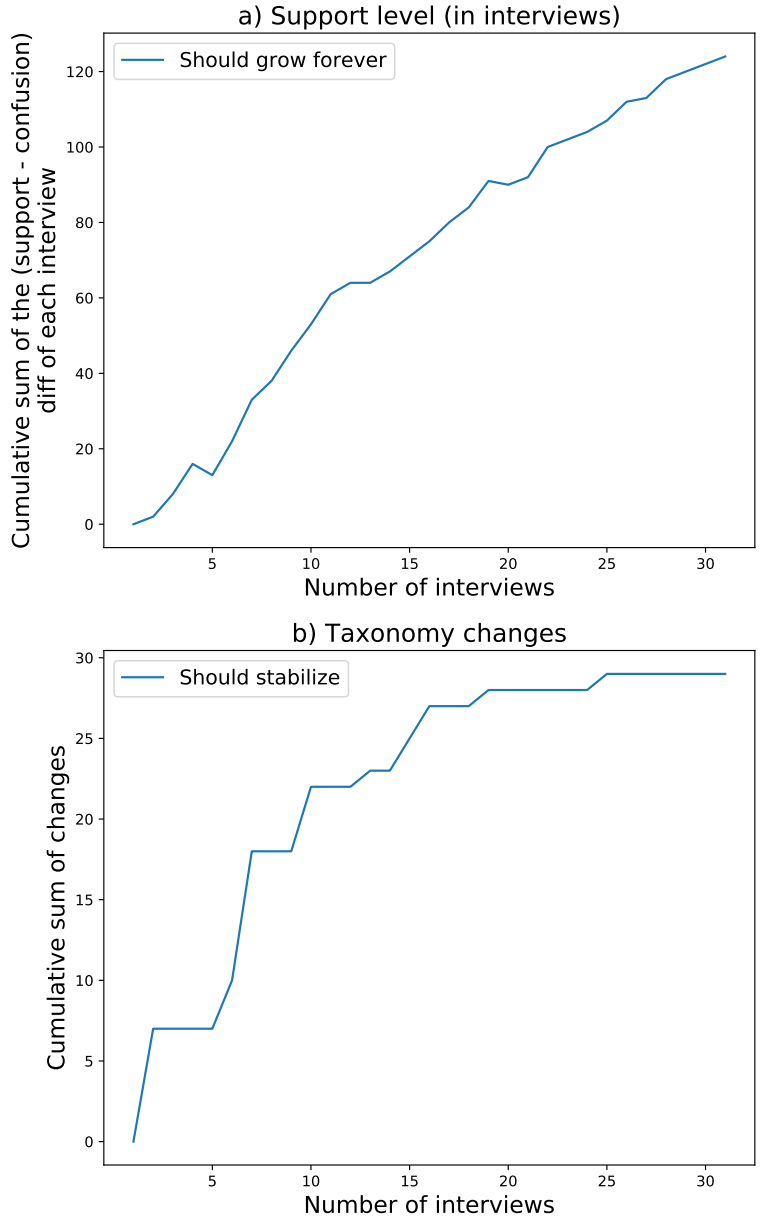

Fig. 2. Metrics of theoretical saturation for the resonance analysis

interviewees. We incorporated report improvements in descriptions presented in our online digest of organizational structures ${ }^{2}$. We provide the list of supports and confusions, plus a summary of actions as supplementary material.

One example of a fragment (I44) coded as confusion that led to a taxonomy change: "I think it's an in-house opensource platform, because although we built it on top of cloud services, AWS in particular, our ecosystem is mostly open-source, based on Kubernetes and its ecosystem." Before this comment, we considered that open-source platforms were installed and ran in physical infrastructure only. Then we noted the following memo [8] during analysis: "Good point... maybe we have to expand the scope of the in-house platform (...) when this use of the cloud is merely the use of virtual machines; the company is still building/installing/managing something on its own."

Therefore, to address this confusion, we took the action of renaming the supplementary property "in-house opensource platform" to "in-house-administered open-source platform." Adding the word "administered" suggests what matters is the company administering the open-source platform regardless of whether it is installed in a physical server or in a virtual machine provided by a cloud vendor.

Another notable change in the taxonomy was renaming "Platform team" to "API-mediated departments." We had confusions in four interviews (I40, I42, I50, I52) due to

2. ccsl.ime.usp.br/devops/2021-06-20/structures-digest.html 


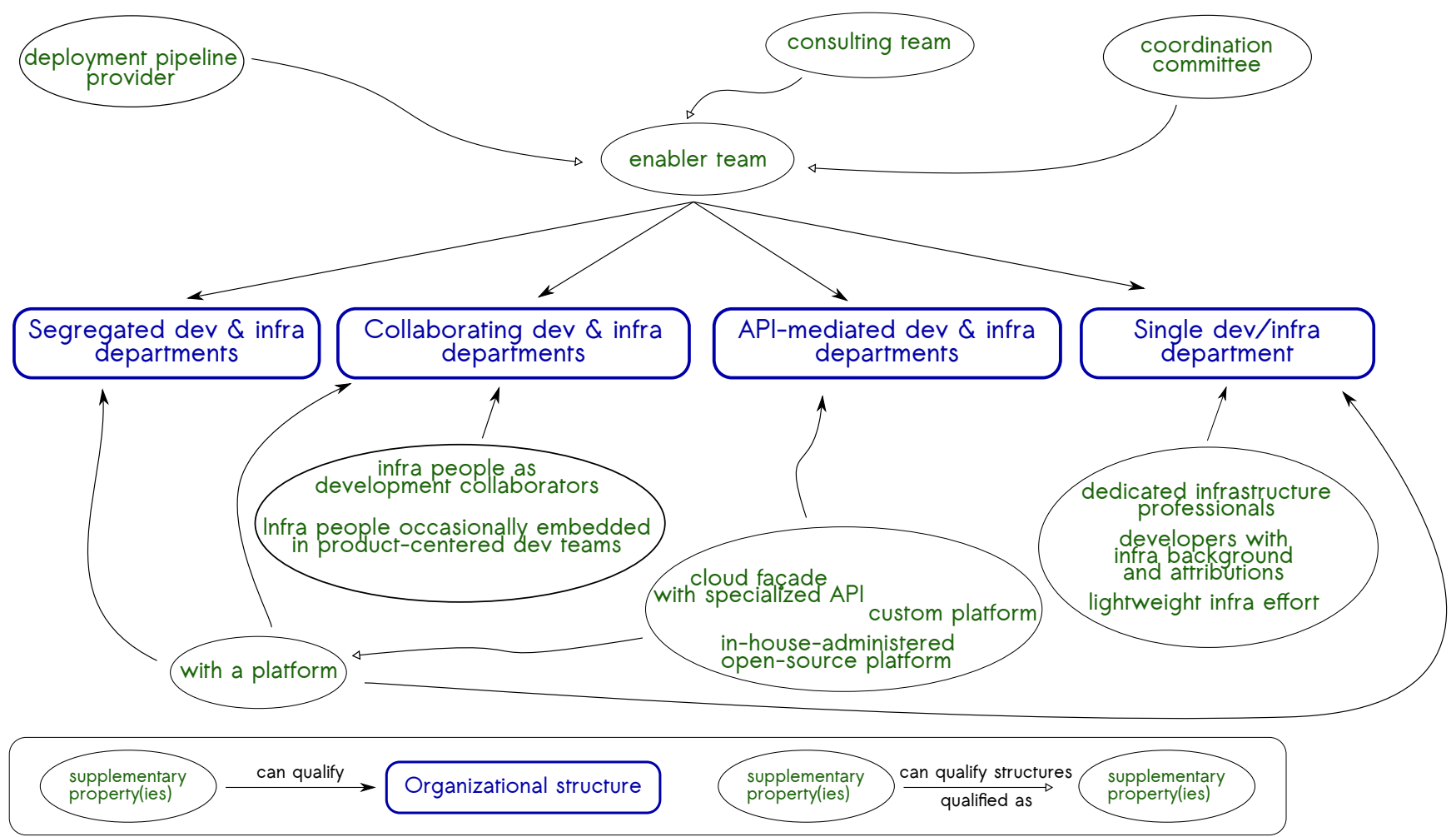

Fig. 3. The high-level view of our taxonomy after the refinement process

the polymorphic meanings of the terms "platform" and "platform team." Moreover, the current name reflects the structure better, since the platform team is just one of its interplaying teams.

Figure 3 presents the consolidated version of our taxonomy, as evolved from the refinements. The figure shows the taxonomy's high-level elements: its structures and their associated supplementary properties. We provide all the versions of the high-level view of our taxonomy as supplementary material.

\section{EXPLAINING ORGANIZATIONAL STRUCTURES}

Now we present, in Tables 3, 4, 5, and 6, the 46 discovered strong codes grouped by organizational structure. Each strong code is preceded by its identifier (e.g., SC02) and the amount of supporting interviews.

TABLE 3

Strong codes for segregated dev \& infra departments

\begin{tabular}{ll}
\hline & Consequences \\
\hline SC01 (5) & Devs lack autonomy and depend on ops \\
SC02 (4) & Low delivery performance (queues and delays) \\
SC03 (3) & Friction and blaming games between devs and infra \\
\hline
\end{tabular}

During the coding process, we linked a few codes to supplementary properties of our taxonomy. The strong codes having such attribution are SC15 (associated with "dedicated infrastructure professionals") and SC22 (associated with "developers with infra background and attributions").

We also report, in Table 7, the total number of codes (not only strong codes) found per code class (6C label vs.
TABLE 4

Strong codes for collaborating dev \& infra departments

\begin{tabular}{ll}
\hline & Conditions \\
\hline SC04 (5) & $\begin{array}{l}\text { Enough infra people to align with dev teams } \\
\text { Top management support }\end{array}$ \\
\hline SC05 (3) & Causes \\
\hline SC06 (4) & $\begin{array}{l}\text { In a non-large company / with few products, } \\
\text { it is easier to be collaborative }\end{array}$ \\
SC07 (3) & $\begin{array}{l}\text { Trying to avoid the delivery bottleneck } \\
\text { Bottom-up initiative with later } \\
\text { top-management support }\end{array}$ \\
& Consequences \\
\hline SC09 (6) & $\begin{array}{l}\text { Growing interaction inter-areas } \\
\text { (e.g., knowledge sharing) }\end{array}$ \\
SC10 (5) & $\begin{array}{l}\text { Precarious collaboration (ops overloaded) } \\
\text { Discomfort/frustration/friction/inefficiency with } \\
\text { blurred responsibilities (people don't know what } \\
\text { to do or what to expect from others) } \\
\text { SC12 (3) }\end{array} \begin{array}{l}\text { Waiting (hand-offs), infra still a bottleneck } \\
\text { Automation supports collaboration }\end{array}$ \\
SC13 (3) Contingencies \\
\hline SC14 (3) & $\begin{array}{l}\text { Giving more autonomy to devs } \\
\text { (in staging or even production) }\end{array}$ \\
&
\end{tabular}

organizational structure). One example of a code that is not a strong code, an avoidance reason for single departments found in I55 and I58, is "specialized knowledge brings scale gains." We list all the codes in the supplementary material.

\subsection{Covariance analysis}

Covariance, part of the $6 \mathrm{C}$ analysis, means the occurrence of one code correlates with the occurrence of another code [43], [45]. Covariance analysis reveals codes commonly supported by multiple interviews, providing more insight 
TABLE 5

Strong codes for single dev \& infra departments

\begin{tabular}{ll}
\hline & Conditions \\
\hline SC15 (3) & Enough ops for each dev team \\
\hline & Causes \\
\hline SC16 (10) & $\begin{array}{l}\text { Startup scenario (small, young, weak infra scalability } \\
\text { requirements, business focus, use of cloud services } \\
\text { to limit costs) }\end{array}$ \\
SC17 (6) & $\begin{array}{l}\text { Cloud services decrease the need of infra \& ops staff } \\
\text { Delivery velocity, agility, critical project }\end{array}$ \\
\hline SC18 (3) & Avoidance reasons \\
\hline SC19 (4) & $\begin{array}{l}\text { Not suitable for applying corporate } \\
\text { governance \& standards } \\
\text { Sore (3) }\end{array}$ \\
& $\begin{array}{l}\text { high infra professionals salary, underused } \\
\text { infra professionals }\end{array}$ \\
\hline & Consequences \\
\hline SC21 (9) & $\begin{array}{l}\text { No [infra] defaults across teams: freedom, } \\
\text { but possibly leading to duplication of efforts and } \\
\text { high maintenance costs }\end{array}$ \\
\hline Contingencies \\
\hline SC22 (3) & Improve infra skills in-house, including tech talks \\
\hline
\end{tabular}

into theory building. We define a strong covariance group as a group of at least three codes related to a set of at least three sources. For example, codes A, B, and C are in the same strong covariance group when all of them are supported by interviews 1, 2, and 3.

Following this definition, we found: 17 strong groups with 3 codes linked to a 3 source-set; 1 strong group with 4 codes linked to a 3 source-set; 1 strong group with 5 codes linked to a 3 source-set; and 2 strong groups with 3 codes linked to a 4 source-set. Table 8 describes the last four strong covariance groups, which are the strongest ones since they have more codes or more sources.

\section{Discussion}

RQ1 is about why different organizations adopt different structures for development and infrastructure professionals. Conditions and causes in the 6C analysis point in this direction. For example, a startup, still struggling to validate its value proposition, is not in a moment to be so cautious about infrastructure requirements. Therefore, such a startup scenario (SC16) usually leads to the adoption of single departments, with developers taking care of infrastructure concerns. After scalability and other infrastructure concerns gain relevance for the company, and the company increases its portfolio with multiple products to multiple clients (SC32), API-mediated departments is seen as a path to overcome existing delivery bottlenecks (SC27), and this promise seems to be fulfilled (SC39). Collaborative departments requires certain parity in the ratio of development to infrastructure people (SC15) to increase its success possibility. Therefore, having a low number of infrastructure professionals and a hierarchy culture (SC28), hampering direct contact between departments on a daily basis, are forces pushing to API-mediated departments.

RQ2 inquires about the drawbacks and mitigations for each structure. Avoidance reasons, consequences, and contingencies in the $6 \mathrm{C}$ analysis provide us answers. Although planned to increase direct cooperation (SC09), collaborative departments may present side effects since responsibilities
TABLE 6

Strong codes for API-mediated dev \& infra departments

\begin{tabular}{ll}
\hline & Conditions \\
\hline SC23 (8) & $\begin{array}{l}\text { Medium to large sized company } \\
\text { Top-down initiatives/sponsorship }\end{array}$ \\
SC24 (5) & Upfront investment \\
SC26 (3) & Requires coding skills from infra people \\
\hline & Causes \\
\hline SC27 (8) & $\begin{array}{l}\text { Delivery bottleneck in infra management } \\
\text { Compatible with existing rigid structures (low impact } \\
\text { on organogram) / Only a few people needed for a }\end{array}$ \\
platform team \\
SC29 (4) & $\begin{array}{l}\text { Fosters continuous delivery } \\
\text { A hero or visionary (hero culture) }\end{array}$ \\
SC30 (4) & $\begin{array}{l}\text { Emerged as best solution; other initiatives } \\
\text { nC31 (4) so fruitful }\end{array}$ \\
SC32 (3) & $\begin{array}{l}\text { Multiple products / multiple dev teams / multiple } \\
\text { clients (requires high delivery performance) }\end{array}$ \\
& Consequences \\
\hline SC33 (8) & $\begin{array}{l}\text { Interaction (devs x platform team) to: support devs, } \\
\text { make things work, and demand new capabilities } \\
\text { from the platform } \\
\text { The platform provides common mechanisms } \\
\text { (e.g., scaling, billing, observability, monitoring) }\end{array}$ \\
SC34 (7) & $\begin{array}{l}\text { Promotes continuous delivery, agility, and } \\
\text { faster changes } \\
\text { Devs responsible for infra architecture / concerns } \\
\text { (e.g., NFR) }\end{array}$ \\
SC35 (4) & $\begin{array}{l}\text { Platform team provides consulting and documentation } \\
\text { to devs } \\
\text { Adding devs do not require adding [proportionally] } \\
\text { more infra people } \\
\text { SC36 (4) }\end{array}$ \\
Eliminated previous bottleneck \\
SC45 (3)
\end{tabular}

become blurred (SC11). Some interviewees expressed concerns about governance and technological standardization. We found that multiple single departments in mid-sized or large companies can be an obstacle to such standardization (SC19). If this is a concern ${ }^{3}$, the company may prefer to adopt API-mediated departments. A peculiar disadvantage of API-mediated departments is that programmers are less likely to be aware of the infrastructure (SC42), which may not be a disadvantage for the company, but possibly for the developers' careers. In this context, we witnessed discussions about to which degree to expose infrastructure details to developers (SC46) and strategies to communicate how to use the platform, such as personalized consulting and mass communication (SC37). These discussions also emerged from situations with programmers overly relying

3. We interviewed one very large company (I38) adopting single departments that were not concerned with standardization: fostering an inner "free market" of solutions was an innovation strategy. 
TABLE 7

Amount of codes found per code class

\begin{tabular}{|c|c|c|c|c|}
\hline Characteristics & 5 & 4 & 6 & 2 \\
\hline Conditions & 0 & 4 & 6 & 3 \\
\hline Causes & 5 & 9 & 25 & 8 \\
\hline Avoidance reasons & 1 & 1 & 3 & 7 \\
\hline Consequences & 7 & 16 & 32 & 13 \\
\hline Contingencies & 1 & 9 & 13 & 18 \\
\hline
\end{tabular}

TABLE 8

Strong covariance groups

\begin{tabular}{lll}
\hline $\begin{array}{l}\text { Group } \\
\text { reference }\end{array}$ & $\begin{array}{l}\text { Strong } \\
\text { codes }\end{array}$ & $\begin{array}{l}\text { Supporting } \\
\text { interviews }\end{array}$ \\
\hline G1 & SC27, SC33, SC34, SC37 & I57, I59, I62 \\
G2 & SC10, SC24, SC27, SC30, SC31 & I40, I41, I48 \\
G3 & SC27, SC33, SC34 & I48, I57, I59, I62 \\
G4 & SC10, SC24, SC27 & I40, I41, I48, I49 \\
\hline
\end{tabular}

on the platform, ignoring the bare minimum of infra management they should master (SC44).

In this way, the association of concepts of our taxonomy to $6 \mathrm{C}$ codes provides explanations about the structures' phenomenon. Such a new explanatory dimension enables scholars to understand structures more deeply and better equip practitioners to discuss them and make decisions.

Unfortunately, the codes of different $6 \mathrm{C}$ labels were not evenly distributed across the structures of our taxonomy. In particular, we found more strong codes for API-mediated departments, and we had only three contingency strong codes. We identified many more contingencies (38 in total), but most of them were supported by only one interview. This may point to a lack of structure awareness by the community, so each company tries to handle the problems in different ways. It could also reflect the peculiarities of the organizations, but evaluating the raw data, that does not seem to be the case. For example, "Playground area so that devs can learn infra" (I50) appears to be a contingency that could be applied to many more organizations. Another interpretation points to the analysis strategy: we considered a contingency as "a solution to one problem," which splits a common solution into different codes.

The largest class of strong codes (6C label and structure) we found was consequences for API-mediated departments (32 consequences). This suggests that this structure may have more predictable outcomes than the others. Also, from the eight codes within the strong covariance groups, seven are related to API-mediated departments. This also puts API-mediated departments as a more understandable phenomenon. Interestingly, the presence of a disadvantage of collaborative departments (SC10) within strong covariance groups $(G 2, G 4)$ shows a motivation for adopting APImediated departments.

The structure for which we found more codes suggesting failure scenarios was collaborative departments (SC10, $\mathrm{SC} 11, \mathrm{SC} 12)$. We consider it may be easier for large organizations with segregated structures trying to move first to collaborative departments. However, having a limited number of infrastructure professionals and not giving them enough budget to interact with developers are recurring factors leading to overload (SC10). Such a situation makes professionals forget the DevOps initiative and revert to the previous siloed style of work. Giving more autonomy to devs (SC14) is an attempt to handle this scenario. Moreover, we found more examples of collaborative departments in which the infrastructure remained as a bottleneck in the delivery path (SC12). This reinforces our previous finding that there is no correlation between collaborative departments and delivery performance [26].

Finally, an additional benefit of our theory, provided by its building process, is offering a taxonomy with objective terms. This concern, for example, led us to replace "silo" (a metaphor) with "segregated" in our refinement process. Such objectiveness is valuable considering the amount of energy and time practitioners take in discussing again and again what DevOps is ${ }^{4}[3]$.

\section{QUALITY CRITERIA}

The quality criteria we pursued are the ones defined by Guba [37] for naturalist inquiries: credibility (how plausible or true the findings are); dependability (methodology applied consistently); confirmability (opportunities for correcting research bias); and transferability (generalizability). Such criteria are widely used in Grounded Theory (GT) studies [6], [33], [50], [51]. For meeting such criteria, we applied the following treatments [50]:

- Providing a chain of evidence (available at our supplementary material), which contributes to credibility and dependability.

- Collecting interviewees' opinions on the results, i.e., member check (Section 7.1), which contributes to credibility and confirmability.

- Having a diverse selection of participants (see Section 3.1), including the triangulation with development and infrastructure staff within some organizations, which contributes to transferability;

- Triangulating among coauthors through a review process (Section 3.5) and methodology revision by a coauthor experienced in qualitative methods [52], which contribute to confirmability.

- Providing quantified evidence of saturation (Section 3.6), which contributes to dependability (this is an additional quality measure, not standard in other works).

- Reporting only codes confirmed by multiple participants (strong codes), which contributes to credibility and transferability.

\subsection{Member check}

Grounded Theory aims to formulate relevant theories for practitioners, so it is crucial to investigate whether findings make sense to them [22]. However, it is opportune to highlight that the goal of our member check is to assess only the theory resonance [22] with participants and not to validate the theory itself. Participants are not obliged to

4. See, for example, the "Chef Style DevOps Kungfu" talk. 
verify whether the abstractions risen from diverse data are conceptually adequate [53]. Therefore, readers should consider member check together with other quality treatments.

We performed member check by collecting feedback on our results from the participants using online surveys, which we provide as supplementary material. For each structure, we prepared one form listing its corresponding strong codes. For each strong code, the respondent had to tick one option within the following Likert scale: "true", "usually true", "no correlation / I don't know", "usually false", "false". We also left a field for general comments. Because opining on all strong codes would take too long, we asked each participant to answer only one form as a strategy to increase the response rate. Nonetheless, they were free to answer all the forms if they so wished.

We sent the feedback requests in two rounds (first and second phase participants). We received responses from 18 of the 68 participants. We received 5 responses for the segregated departments form, 6 for the collaborating departments form, 5 for the API-mediated departments form, and 9 for the single departments form. From the 272 manifested opinions on strong codes, 166 were favorable $(61 \%$ of the interviewees considered them to be true or usually true), while only 45 were unfavorable (usually false or false). In particular, no strong code received only unfavorable feedback. This result suggests a good resonance of the participants with our theory.

According to a respondent's comment, disagreements related to single departments may relate to possible interpretations depending on single departments having infrastructure specialists. Indeed, in the forms, we did not associate SC15 and SC22 to their respective supplementary properties (see Section 5), which possibly jeopardized respondents' reasoning. This issue was fixed for the second round of feedback requests.

\subsection{Generalizability}

Although we cannot claim generalizability with statistical confidence, the employed methods and the taken sample provide some relative generalizability to our theory (at least more than case studies [36] usually provide).

We took a diverse sample. Considering semi-structured interviews in the first and second research phases, we interviewed 20 companies with more than 1,000 employees, 17 with between 200 and 1,000 employees, and 17 with less than 200 employees. We interviewed people in 8 countries. The company domains also varied wildly.

In the second research phase, we applied our taxonomy in conversations with professionals working in companies that did not provide data to the classification construction in the first phase. In the context of our resonance analysis, among the interviews in new companies, we had 99 codes of support and 25 codes of confusion (four times more support than confusion), which contributes to showing the generalizability of our theory. In addition, confusion codes started to rarefy at the last interviews since we used confusions to improve the theory. In the $6 \mathrm{C}$ analysis, we found 15 characteristics (of 17 characteristics codes) that define the organizational structures in 15 new companies ( $88 \%$ of the new interviewed companies), which also contributes to show that our theory applies to these new contexts.
We also were cautious about the diversity of the interviewees' roles. Only five $(13 \%)$ of the interviewees had an infrastructure role in the first research phase. In the second phase, we were able to improve this situation. From the 31 interviewees in the second phase, 14 of them (nearly half) had an infrastructure role. Since, for the second phase, we looked for people with more in-house experience, so they could answer our "why questions," we ended up also interviewing a larger fraction of managers $(30 \%$ vs. $61 \%$ of managers).

The $6 \mathrm{C}$ analysis also provided more evidence for some findings of the first phase. In particular, API-mediated departments still seems to be a promising path to achieve high delivery performance in comparison with the other structures. Such results now concur with results of other independent studies [6], [27]. We heard of bottlenecks in the delivery path, especially in the segregated and the collaborating departments structures, while some interviewees claimed that the API-mediated structure removed the bottleneck previously existing. Another reinforced findings is that single departments is more associated with small organizations, while API-mediated departments is not.

In summary, the points strengthening our theory's generalizability are: (i) diversity of professional and company profiles in our sample; (ii) applying our taxonomy to scenarios that did not feed its initial version; (iii) gathering evidence of support for the theory among new companies; and (iv) gathering evidence corroborating preliminary findings.

\section{THREATS TO VALIDITY}

As usual to taxonomical theories [22], our work does not provide probabilistic predictions in terms of dependent and independent variables. As usual to grounded theories [8], some factors (such as interviewees anonymity and unavoidable subjectivity in analysis) make the research not fully replicable. In particular, anonymity is a trade-off with the potential number of interviewees and goes along with ethical research [54]; also, interviewing the same person again does not necessarily yield the same results.

Given cost considerations, the review process has limitations. Reviewing authors read only some transcriptions excerpts: whenever needed during reviews, we searched for these relevant excerpts to support our discussions. Our research data corresponds to people's views and opinions, which may drift from objective reality. Therefore, an observational research approach would be desirable [22]. However, our research questions are too abstract to grasp only by observation, even meeting observations, without further conversations with the observed people. Therefore, observational research with the same goal as ours would be much more expensive, if at all feasible, especially involving as many organizations as we did.

Responses were dependent on how we presented the taxonomy, which interviewees could misunderstand. A mitigation for this issue was analyzing how to improve the taxonomy presentation for the subsequent sessions.

\section{Conclusion}

Our research provides a theory on how software-producing companies organize their development and infrastructure 
workforce according to different organizational structures: segregated departments, collaborative departments, APImediated departments, and single department. Some of these structures have relevant variations represented in our taxonomy (e.g., single departments may or may not encompass staff dedicated to infrastructure). We found why different organizations adopt (or do not adopt) different structures and the conditions leading to this choice (e.g., API-mediated departments are pursued to address delivery bottlenecks; single departments are avoided for its unsuitability in enforcing corporate standards; and collaborating departments require proportional infra people per development team). We also found that each structure has drawbacks and how organizations deal with such disadvantages (e.g., collaborating departments may lead to conflicts due to blurred responsibilities; tuning the platform abstraction level may prevent developers from over-relying on the platform as magical). A notable result is that we found many contingencies, but only a few shared among different organizations, which can be a consequence of the lack of awareness of organizational structure patterns in the software industry.

This work has implications for practice. By increasing the awareness of organizational structures in the community, practitioners can make more informed decisions on structure selection and drawback handling. Moreover, having an up-to-date view of what "other companies are doing" is always a relevant input for practitioners' decision-making. An example is that practitioners can relate observed problems in their organizations to expected consequences under our theory. Such explicit associations can avoid hours of unfruitful discussions, possibly assuming the problem to be "something that only happens here."

This work also has implications for scholars. Professors can update their understanding of the software production phenomenon based on concepts and relations grounded on the current behavior of the software industry. Thus, professors can update their software engineering classes accordingly. A secondary and methodological contribution of our work for developing new grounded theories is providing an objective technique to detect theoretical saturation, which is rarely seen in the literature.

Finally, grounded theories can always be adapted according to the discovery of new instances of the phenomenon. Therefore, further research on the topic is welcome, especially considering that, usually, software engineering theories (as social theories) are not proven to be true. In particular, observational studies would be desirable to strengthen (or dispute) our theory. Given the existence of other taxonomies in the DevOps context, promising future work is conciliating them in a unified model. Such unification may also demand methodological advances: e.g., how to merge taxonomies and validate such an integration? We also believe many more insights and discussions can be derived from our strong codes, be it in academic or practitioners forums. In particular, a relevant implication for researchers is that each strong code could be submitted to validation studies or, at least, be a starting point to new studies.

\section{ACKNOWLEDGMENTS}

We thank the support of the Brazilian Service of Federal Data Processing (Serpro), CNPq proc. 465446/2014-0, CAPES - Finance Code 001, and FAPESP procs. 14/50937-1, 15/24485-9, and 2019/12743-4.

\section{RefERENCES}

[1] “ITIL $4^{\text {th }}$ edition, glossary," 2019, https://purplegriffon. com/downloads/resources/itil4-foundation-glossary-january2019.pdf, accessed on Nov 2021.

[2] J. Fulmer, "What in the world is infrastructure," PEI Infrastructure Investor, vol. 1, no. 4, pp. 30-32, 2009.

[3] L. Leite, C. Rocha, F. Kon, D. Milojicic, and P. Meirelles, "A survey of DevOps concepts and challenges," ACM Computing Surveys, vol. 52, no. 6, pp. 127:1-127:35, 2019.

[4] K. Nybom, J. Smeds, and I. Porres, "On the impact of mixing responsibilities between devs and ops," in International Conference on Agile Software Development, ser. XP 2016. Springer International Publishing, 2016, pp. 131-143.

[5] M. Shahin, M. Zahedi, M. A. Babar, and L. Zhu, "Adopting continuous delivery and deployment: Impacts on team structures, collaboration and responsibilities," in Proceedings of the 21st International Conference on Evaluation and Assessment in Software Engineering, ser. EASE'17. ACM, 2017, pp. 384-393.

[6] D. Lopez-Fernandez, J. Diaz, J. Garcia-Martin, J. Perez, and A. Gonzalez-Prieto, "Devops team structures: Characterization and implications," IEEE Transactions on Software Engineering, 2021.

[7] R. W. Macarthy and J. M. Bass, "An empirical taxonomy of DevOps in practice," in 2020 46th Euromicro Conference on Software Engineering and Advanced Applications (SEAA), 2020, pp. 221-228.

[8] B. Glaser and A. Strauss, The discovery of grounded theory: strategies for qualitative research. Aldine Transaction, 1999.

[9] S. Gregor, "The nature of theory in information systems," MIS quarterly, pp. 611-642, 2006.

[10] J. Stoner and R. E. Freedman, Administração. Prentice-Hall, 1995.

[11] L. Donaldson, "Teoria da contingência estrutural," in Handbook de estudos organizacionais. Atlas, 1999.

[12] R. H. Hall, Organizações, estruturas e processo. Prentice-Hall, 1984.

[13] N. Oliveira and N. Takahashi, "Organizational structure, format shape design and architecture," in Automated organizations: Development and structure of the modern business firm. Springer, 2012.

[14] M. E. Conway, "How do committees invent," Datamation, vol. 14, no. 4, pp. 28-31, 1968.

[15] F. C. Lunenburg, “Organizational structure: Mintzberg's framework," International journal of scholarly, academic, intellectual diversity, vol. 14, no. 1, 2012.

[16] D. E. Yeatts and C. Hyten, High-Performing Self-Managed Work Teams: A Comparison of Theory to Practice. Sage Publications, 1998.

[17] D. S. Pugh, D. J. Hickson, and C. R. Hinings, "An empirical taxonomy of structures of work organizations," Administrative Science Quarterly, vol. 14, no. 1, 1969.

[18] M. E. Sosa, S. D. Eppinger, and C. M. Rowles, "The misalignment of product architecture and organizational structure in complex product development," Management Science, vol. 50, no. 12, 2004.

[19] C. B. Seaman and V. R. Basili, "Communication and organization: an empirical study of discussion in inspection meetings," IEEE Transactions on Soft. Engineering, vol. 24, no. 7, pp. 559-572, 1998.

[20] J. Herbsleb and J. Roberts, "Collaboration in software engineering projects: A theory of coordination," in International Conf. on Information Systems 2006 Proceedings, ser. ICIS 2006, 2006, pp. 553-568.

[21] N. Nagappan, B. Murphy, and V. Basili, "The influence of organizational structure on software quality," in 2008 ACM/IEEE 30th International Conference on Software Engineering, 2008, pp. 521-530.

[22] P. Ralph, "Toward methodological guidelines for process theories and taxonomies in software engineering," IEEE Transactions on Software Engineering, vol. 45, no. 7, pp. 712-735, 2019.

[23] M. Skelton and M. Pais, “Devops topologies," 2013, https://web. devopstopologies.com/, accessed on Nov 2021.

[24] - Team Topologies: Organizing business and technology teams for fast flow. IT Revolution Press, 2019.

[25] A. Mann, M. S. A. Brown, and N. Kersten, "2018 State of DevOps Report," 2018, https://puppet.com/resources/whitepaper/2018state-of-devops-report, accessed on Jul 2019. 
[26] L. Leite, G. Pinto, F. Kon, and P. Meirelles, "The organization of software teams in the quest for continuous delivery: A grounded theory approach," Information and Software Technology, vol. 139, p. 106672, 2021.

[27] A. Brown, M. Stahnke, and N. Kersten, "2020 State of DevOps Report," 2020, https://www2.circleci.com/2020-state-of-devopsreport.html, accessed on Dec 2021

[28] L. Leite, F. Kon, G. Pinto, and P. Meirelles, "Building a theory of software teams organization in a continuous delivery context," in 42nd International Conference on Software Engineering Companion, ser. ICSE '20 Companion, 2020, pp. 294-295.

[29] L. Leite, G. Pinto, F. Kon, and P. Meirelles, "Platform teams: An organizational structure for continuous delivery," in IEEE/ACM 42nd International Conference on Software Engineering Workshops, ser. ICSEW'20, 2020, pp. 505-511.

[30] N. Forsgren, M. A. Rothenberger, J. Humble, J. B. Thatcher, and D. Smith, "A taxonomy of software delivery performance profiles: Investigating the effects of DevOps practices," in AMCIS 2020 Proceedings, no. 8, 2020.

[31] F. M. A. Erich, C. Amrit, and M. Daneva, "A qualitative study of DevOps usage in practice," Journal of Software: Evolution and Process, vol. 29, no. 6, 2017.

[32] K.-J. Stol, P. Ralph, and B. Fitzgerald, "Grounded theory in software engineering research: A critical review and guidelines," in 2016 IEEE/ACM 38th International Conference on Software Engineering, ser. ICSE '16, 2016, pp. 120-131.

[33] M. Jovanović, A. Mas, A.-L. Mesquida, and B. Lalić, “Transition of organizational roles in agile transformation process: A grounded theory approach," Journal of Systems and Software, vol. 133, 2017.

[34] K.-J. Stol and B. Fitzgerald, "The ABC of software engineering research," ACM Transac. on Software Engineering and Methodology, vol. 27, no. 3, 2018.

[35] W. C. Adams, "Conducting semi-structured interviews," in Handbook of Practical Program Evaluation, 3rd ed. Jossey-Bass, 2010.

[36] R. K. Yin, Case Study Research, Design and Methods, 4th ed. Sage Publications, 2009.

[37] E. Guba, "Criteria for assessing the trustworthiness of naturalistic inquiries," Educational Technology Research and Development (ECTJ), vol. 29, pp. 75-91, 1981.

[38] D. G. Sirmon, M. A. Hitt, R. D. Ireland, and B. A. Gilbert, "Resource orchestration to create competitive advantage: Breadth, depth, and life cycle effects," Journal of management, vol. 37, no. 5, pp. 1390-1412, 2011.

[39] L. T. Pinfield, "A field evaluation of perspectives on organizational decision making," Administrative Science Quarterly, vol. 31, no. 3, pp. 365-388, 1986.

[40] P. A. Anderson, "Decision making by objection and the Cuban missile crisis," Administrative Science Quarterly, vol. 28, no. 2, pp. 201-222, 1983.

[41] I. Steinmacher, T. U. Conte, C. Treude, and M. A. Gerosa, “Overcoming open source project entry barriers with a portal for newcomers," in Proceedings of the 38th International Conference on Software Engineering, ser. ICSE '16. ACM, 2016, pp. 273-284.

[42] H.-F. Hsieh and S. E. Shannon, "Three approaches to qualitative content analysis," Qualitative health research, vol. 15, no. 9, pp. 1277-1288, 2005.

[43] B. Glaser, Theoretical Sensitivity: Advances in the Methodology of Grounded Theory. The Sociology Press, 1978.

[44] J. Saldaña, The coding manual for qualitative researchers. Sage, 2015.

[45] R. Hoda, J. Noble, and S. Marshall, "The impact of inadequate customer collaboration on self-organizing agile teams," Information and software technology, vol. 53, no. 5, pp. 521-534, 2011.

[46] G. van Waardenburg and H. van Vliet, "When agile meets the enterprise," Information and Software Technology, vol. 55, no. 12, pp. 2154-2171, 2013.

[47] B. Glaser, The grounded theory perspective III: Theoretical coding. The Sociology Press, 2005, pp. 70.

[48] C. A. Hernandez, "Theoretical coding in Grounded Theory methodology," Grounded Theory Review, vol. 8, no. 3, 2009.

[49] M. Waterman, J. Noble, and G. Allan, "How much up-front?: A grounded theory of agile architecture," in 2015 IEEE/ACM 37th IEEE International Conference on Software Engineering, ser. ICSE '15, 2015, pp. 347-357.

[50] M. G. Waterman, "Reconciling agility and architecture: a theory of agile architecture," Ph.D. dissertation, Victoria University of Wellington, 2014.
[51] M. Shahin and M. A. Babar, "On the role of software architecture in DevOps transformation: An industrial case study," in Proceedings of the International Conference on Software and System Processes, ser. ICSSP '20. ACM, 2020, pp. 175-184.

[52] C. Melo, "Productivity of agile teams: an empirical evaluation of factors and monitoring processes," Ph.D. dissertation, University of São Paulo, 2015.

[53] B. G. Glaser, "Conceptualization: On theory and theorizing using grounded theory," International Journal of Qualitative Methods, vol. 1, no. 2, pp. 23-38, 2002.

[54] P. E. Strandberg, "Ethical interviews in software engineering," in International Symposium on Empirical Software Engineering and Measurement 2019, ser. ESEM '19, 2019.

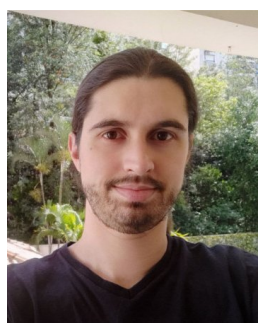

Leonardo Leite received the Bachelor's degree in computer engineering and the MSc degree in Computer Science from the University of São Paulo (USP) in 2010 and 2014. He is currently a $\mathrm{PhD}$ candidate at USP and a software developer at the Brazilian Service of Federal Data Processing (Serpro).

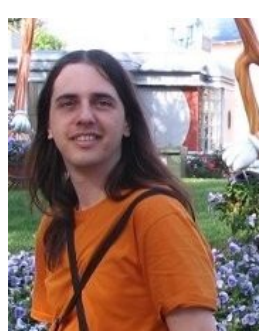

Nelson Lago received the Bachelor's degree in music and the MSc degree in Computer Science from the University of São Paulo (USP) in 2000 and 2004. He is currently the Technical Manager of the USP FLOSS Competence Center, and works both as researcher and IT operations manager.

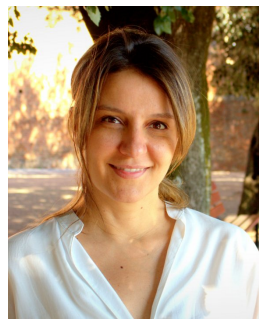

Claudia Melo received the PhD degree in Computer Science from the University of São Paulo (USP) in 2013. Former professor at the University of Brasília (UnB) and head of technology for Latin America at ThoughtWorks. She is now a Director of Software Engineering/Tech Org Design at Loft.

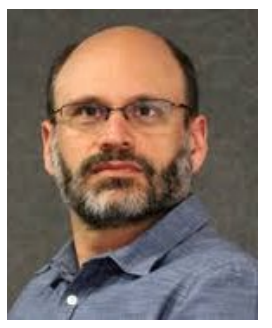

Fabio Kon received the $\mathrm{PhD}$ degree in Computer Science from the University of Illinois Urbana-Champaign in 2000. He is a Full Professor at the University of São Paulo (USP), researching software engineering, agile methods, distributed systems, smart cities, entrepreneurship, and computer music.

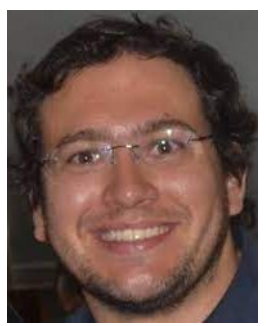

Paulo Meireless received the $\mathrm{PhD}$ degree in Computer Science from the University of São Paulo (USP) in 2013. He is an adjunct professor at the Federal University of ABC (UFABC). He also works as a collaborating researcher at USP, investigating Software Engineering, with a focus in Free Software and DevOps. 